\title{
BJO
}

\section{An unexpectedly low Stratus optical coherence tomography false-positive rate in the non-nasal quadrants of Asian eyes: indirect evidence of differing retinal nerve fibre layer thickness profiles according to ethnicity}

T W Kim, T-W Kim, K H Park, et al.

Br J Ophthalmol 2008 92: 735-739

doi: 10.1136/bjo.2007.129502

Updated information and services can be found at:

http://bjo.bmj.com/content/92/6/735.full.html

\section{These include:}

References This article cites 19 articles, 12 of which can be accessed free at: http://bjo.bmj.com/content/92/6/735.full.html\#ref-list-1

Article cited in:

http://bjo.bmj.com/content/92/6/735.full.html\#related-urls

Email alerting

Receive free email alerts when new articles cite this article. Sign up in the service box at the top right corner of the online article.

Notes

To order reprints of this article go to:

http://bjo.bmj.com/cgi/reprintform

To subscribe to British Journal of Ophthalmology go to:

http://bjo.bmj.com/subscriptions 


\title{
An unexpectedly low Stratus optical coherence tomography false-positive rate in the non-nasal quadrants of Asian eyes: indirect evidence of differing retinal nerve fibre layer thickness profiles according to ethnicity
}

\author{
T W Kim, ${ }^{1}$ T-W Kim, ${ }^{1,2}$ K H Park, ${ }^{1}$ D M Kim ${ }^{1}$
}

\begin{abstract}
Department of Ophthalmology, Seoul National University College of Medicine, Seoul, Korea; ${ }^{2}$ Department of Ophthalmology, Seoul National University Bundang Hospital, Seongnam, Korea

Correspondence to:

Dr T-W Kim, Seoul National University Bundang Hospital, 300, Gumi-dong, Bundang-gu, Seongnam 463-707, Korea; twkim7@snu.ac.kr
\end{abstract}

Accepted 19 March 2008

\section{ABSTRACT}

Aims: To determine the frequency distribution of falsepositive Stratus optical coherence tomography (OCT) results of healthy eyes in terms of peripapillary location. Methods: One eye of each of 137 healthy Korean subjects was included. The false-positive rates (FPRs) relating to retinal nerve fibre layer (RNFL) thickness parameters were determined.

Results: Of the 12 clock-hour positions, the 2 and 4 o'clock positions showed the highest FPRs of $5.1 \%$ and $4.4 \%$, respectively, and 7, 8 and 11 o'clock had the lowest FPRs of $0 \%$ at $<5 \%$ level. At $<1 \%$ level, 2 and 6 o'clock had an FPR of $0.7 \%$, while the FPR was $0 \%$ for all other clock-hours. The FPRs for quadrant thicknesses at $<5 \%$ level were: $0.7 \%$ for temporal; $0 \%$ for superior; $5.1 \%$ for nasal; and $2.2 \%$ for inferior. At $<1 \%$ level, the inferior showed the highest FPR of $0.7 \%$, and the other three quadrants $0 \%$. According to line graph analysis, the 80-120 test point sections had FPRs of $4.4-6.6 \%$, while the $0-40$ and $210-256$ sections were $0-0.7 \%$ at $<5 \%$ level. At $<1 \%$ level, the 40-60, 90-110 and 200-210 sections had $0.7 \%, 190-200$ section $1.5 \%$, and all other sections $0 \%$.

Conclusion: The FPR for the non-nasal region was much lower than expected at $<5 \%$ level. Given that the current OCT normative database is based on a largely non-Asian population, these findings suggest that RNFL thickness profiles may differ according to ethnicity.

Glaucoma is defined by the structural damage to the optic nerve head $(\mathrm{ONH})$ and retinal nerve fibre layer (RNFL), and the consequent visual field loss as determined by perimetry. In glaucoma patients, structural changes detected using stereo disc photography and red-free RNFL photography precede functional damage detected by perimetry. ${ }^{1-3}$ Although stereo disc photography and redfree RNFL photography are established standard tools for evaluating glaucomatous structural damage, they are subjective and associated with high intra- and interobserver variability. ${ }^{4}$

Optical coherence tomography (OCT) is a noninvasive, high-resolution, cross-sectional imaging technique that allows in vivo measurement of tissue thickness. ${ }^{5}$ The Stratus OCT (Carl Zeiss Meditec, Dublin, CA) is a third-generation machine that has a resolution of $8-10 \mu \mathrm{m}$, and is capable of differentiating between healthy eyes and eyes with glaucoma. ${ }^{6-11}$ Recently, normative reference values for RNFL thickness measurements were established through data collection and analysis of 328 normal individuals. ${ }^{12}$ Several studies have demonstrated a high sensitivity and specificity of the Stratus OCT with its internal normative database for diagnosing glaucoma. ${ }^{13} 14$

Our clinical experience suggests that in the Korean population, OCT results in more false positive findings in the nasal sector than in other sectors. We hypothesised that this may reflect an ethnic difference in the RNFL thickness profile. Thus, the purpose of the present study was to determine the frequency distribution of falsepositive Stratus OCT results of healthy eyes, in terms of peripapillary location, in a sample of Korean subjects.

\section{MATERIALS AND METHODS}

Eyes were chosen from a database of subjects examined for glaucoma in the Department of Ophthalmology, Seoul National University Bundang Hospital, between April 2005 and October 2005. The study was approved by the Seoul National University Bundang Hospital institutional review board and conformed with the Declaration of Helsinki. Informed consent was obtained from all subjects.

Prior to the study, all subjects underwent a complete ophthalmic examination, including visual acuity, refraction, slit-lamp biomicroscopy, gonioscopy, Goldmann applanation tonometry, dilated stereoscopic examination of the optic disc, fundus red-free photography, standard automated perimetry (SAP) and Stratus OCT testing. Inclusion criteria were a BCVA of 20/40 or better, spherical refraction less than \pm 5.0 dioptres (D), cylinder correction less than $\pm 3.0 \mathrm{D}$, an open angle on gonioscopy, a clear ocular media that allowed clear visualisation of the optic nerve head and RNFL in the photographs without any focal or diffuse blurring, absence of glaucomatous optic neuropathy (GON), absence of RNFL defect according to red-free RNFL photography, and a normal SAP result. Absence of GON was defined as a cup-disc ratio less than 0.6 , and an intact neuroretinal rim without peripapillary haemorrhages, notches or localised pallor. Subjects with a history of intraocular surgery other than cataract extraction, any evidence of pathological retinal features, or neurological disorders that may affect the RNFL 
thickness were excluded. Pseudophakic eyes were included only when the cataract surgery had been performed at least 1 year before enrolment.

Red-free RNFL photographs were taken using an EOS D60 digital camera (Canon, Utsunomiyashi, Tochigiken, Japan) following maximal pupil dilation. Sixty-degree, wide-angle views of the optic disc, centred between the superior and inferior regions of the RNFL were obtained and reviewed on an LCD monitor.

Stereoscopic disc photographs (SDP) were also acquired with an EOS D60 digital camera (Canon) on the same occasion as red-free fundus photography. Photographs were assessed using an LCD monitor with a stereoscopic viewer (Screen-Vu Stereo Viewer, Berezin Stereo Photography Products, Mission Viejo, CA).

The stereo disc and red-free RNFL photographs were independently evaluated by two observers (TW Kim, T-W Kim) who were blinded to the patient clinical information, and who each classified eyes into one of the following categories: normal versus abnormal for SDP, and no RNFL defect versus presence of RNFL defect as shown by RNFL photography. Eyes classified by both observers as being normal disc and having no RNFL defect were enrolled in the study. Discrepancies between the two observers were resolved by consensus.

Visual-field analysis was performed using the Swedish Interactive Threshold Algorithm (SITA) standard of the Humphrey Field Analyzer II 750 (Carl Zeiss Meditec, Dublin, CA), using the Humphrey 24-2 test pattern. A normal visual field was defined as follows: ${ }^{15}{ }^{16}$ a glaucoma hemifield test within normal limits, a pattern standard deviation with a $p$ value of $\geqslant 5 \%$, and no existence of a cluster of three contiguous points in the pattern standard deviation plot in a single hemifield with a $\mathrm{p}$ value of $<5 \%$. Only reliable (fixation losses, false positives and false negatives all $\leqslant 25 \%$ ) visual fields were included.

\section{Optical coherence tomography}

OCT was performed using a previously described technique ${ }^{5} 1718$ by one examiner. All measurements were taken using the Stratus OCT after pupillary dilation to a minimum diameter of $5 \mathrm{~mm}$. The operator centred the circular scan on the $\mathrm{ONH}$ while the studied eye was fixated (internal fixation technique). Satisfactory quality was defined as (1) good centration on the optic disc and (2) signal strength $\geqslant 6(10=$ maximum $)$. The fast RNFL thickness scan mode was used. With this scan type, during a single scan the RNFL thickness is determined at 256 points covering a set diameter $(3.4 \mathrm{~mm})$ around the centre of the optic disc 3 times. These values are averaged to yield 12 clock-hour thicknesses, four quadrant thicknesses, and a global average RNFL thickness measurement $\left(360^{\circ}\right.$ measure). These values are then compared against a normative database of agematched controls to derive percentile values. The four percentile values included in the OCT software are the top fifth, top 95th, bottom fifth and bottom first percentiles. In addition, OCT provides a line graph which displayed RNFL thickness on the vertical axis and the A-scan location on the horizontal axis. The display of the RNFL thickness along the scanned circle begins temporally at $0^{\circ}$. The RNFL thickness profile is then plotted in a clockwise direction for a right eye and counterclockwise for a left eye. In the line graph presentation, the percentile values derived from the normative database are represented as colour bands.

RNFL thickness parameters evaluated in this study were temporal, superior, nasal and inferior quadrant thicknesses, and thicknesses for each of the 12 clock-hour positions, with the 9 o'clock position temporal, the 12 o'clock position superior, the 3 o'clock position nasal and the 6 o'clock position deemed inferior.

\section{Data analysis}

The frequency distribution of false-positive Stratus OCT results of healthy eyes, in terms of peripapillary location, was determined. A false-positive result was achieved when the measured value was lower than the given percentile of the intrinsic normative database (ie, $<5 \%$ level or $<1 \%$ level). The false-positive rate (FPR) was defined as the number of subjects having false-positive results divided by the total number of subjects included in the study.

The FPRs at both the $<5 \%$ level and the $<1 \%$ level were determined for each RNFL thickness parameter. In addition, the rate of the presence of a segment in the line graph located below the yellow-coloured band (outside the 95\% normal limit) and red-coloured band (outside the 99\% normal limit) was also assessed in 26 sectors, each comprising 10 test points (the 26th sectors included the 251 st-256th test points). For this analysis, the result was considered positive when any part of the line graph involved the given criteria in each sector. The method of defining RNFL defects using the OCT line graph is described elsewhere. ${ }^{14} 19$ The segment determined as a defect in the OCT RNFL thickness profile showed a strong topographic correlation with localised RNFL defects determined by red-free fundus photography, ${ }^{19}$ with a sensitivity of $85.9 \%$ and specificity of $97.4 \%$ for detecting localised photographic RNFL defects. ${ }^{14}$

In cases where both eyes of a subject were eligible for the study, only one eye was chosen, at random, for inclusion.

\section{RESULTS}

\section{Study sample characteristics}

The study involved 137 eyes from 137 healthy subjects who had received ophthalmic examinations to exclude glaucoma. All subjects had family histories of glaucoma, or had been referred
Figure 1 Frequency distribution of false-positive rates (FPRs) for optical coherence tomography measurements of retinal nerve fibre layer thickness according to test point location. The superonasal region (80-120 test points) had the highest FPR of the 256 test points, while the superotemporal $(0-40$ test points) and inferotemporal (210-256 test points) regions had the lowest FPRs, being less than $1 \%$ at the $<5 \%$ level. INF, inferior; NAS, nasal; SUP, superior; TEMP, temporal.

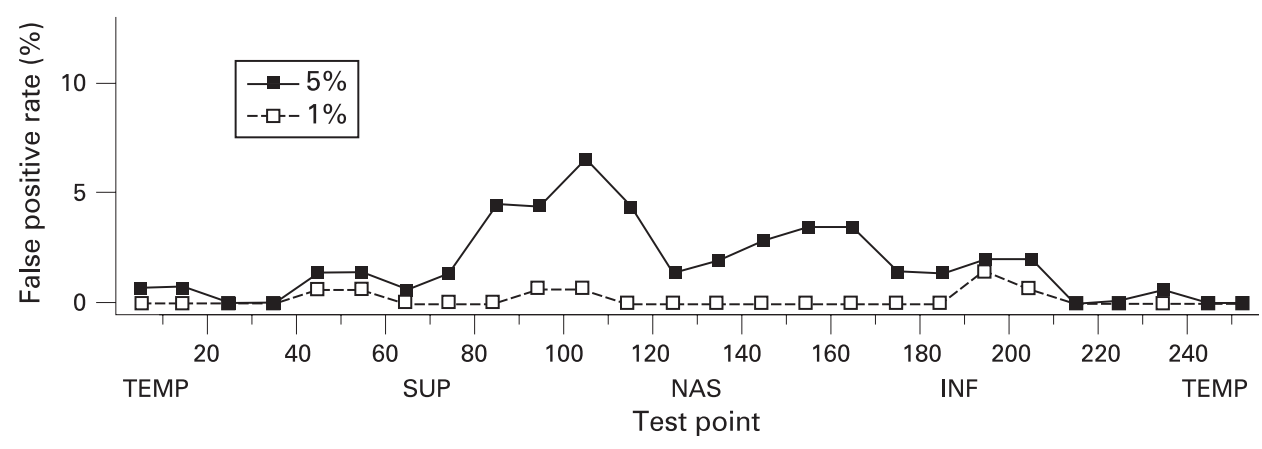


Figure 2 Frequency distribution of the rate of exceeding the 95th percentile of the normative database. The rate was generally high in the non-temporal region, with the highest value being $23.4 \%$ at $240-250$ test points, followed by $22.5 \%$ at $0-10$ and $250-256$ test points. In the nasal region, the values were $5-10 \%$. INF, inferior; NAS, nasal; SUP, superior; TEMP, temporal.

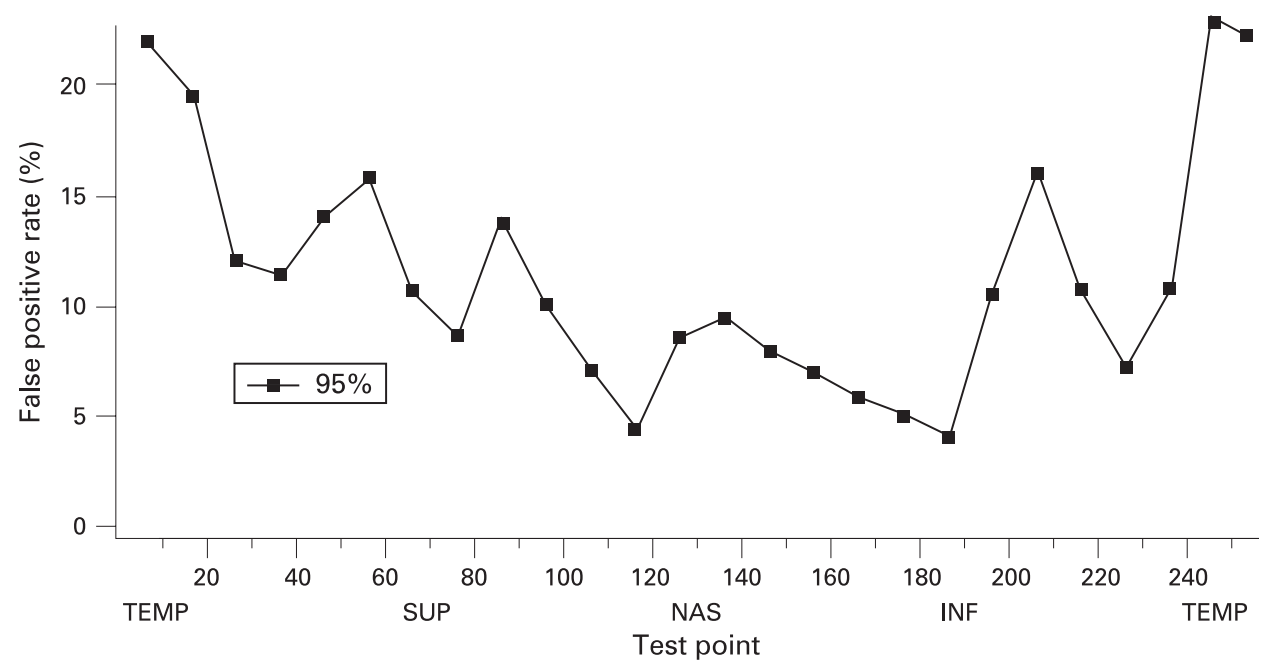

from private clinics or health promotion centres because of suspected optic nerve problems. All individuals were Korean. The sample population comprised 66 females and 71 males. The mean (SD) age was 52.0 (13.4) years (range 19-81 years). The mean (SD) intraocular pressure of the population was 14.4 (3.7) $\mathrm{mm} \mathrm{Hg}$ and the mean (SD) spherical equivalent was -1.03 (2.08) D (table 1). Of 137 eyes, three were pseudophakic, and 134 were phakic eyes.

\section{False positive rates for the 12 clock-hour and quadrant RNFL thicknesses}

The highest FPR for the 12 clock-hour positions was $5.1 \%$ for the 2 o'clock thickness, followed by $4.4 \%$ at 4 o'clock and $2.9 \%$ at 1 o'clock at the $<5 \%$ level. The lowest FPRs were at 7, 8 and 11 o'clock (0\%). At the $<1 \%$ level, the FPR was $0.7 \%$ at 2 and 6 o'clock, and was $0 \%$ at the other clock-hours.

The FPRs at the $<5 \%$ level for the quadrant RNFL thicknesses were $0.7 \%$ for the temporal, $0 \%$ for the superior, $5.1 \%$ for the nasal and $2.2 \%$ for the inferior quadrant. At the $<1 \%$ level, the inferior quadrant had an FPR of $0.7 \%$, while all other quadrants showed $0 \%$ (table 2).

\section{False-positive rates associated with line-graph analysis}

The line-graph analysis showed that the superonasal region (80120 test points) had an FPR of 4.4-6.6\%, which was the highest of all 256 test points at the $<5 \%$ level. The inferonasal region (150-170 test points) showed a relatively high FPR of 3.7\%. The superotemporal (0-40 test points) and inferotemporal (210-256 test points) regions had the lowest FPRs, being less than $1 \%$ at the $<5 \%$ level $(0-0.7 \%)$. At the $<1 \%$ level, the $40-60,90-110$ and 200-210 test-point sections had FPRs of $0.7 \%$, the 190-200 test-point sections had a rate of $1.5 \%$, and all other sections had a rate of $0 \%$ (fig 1 ).

Table 1 Study sample demographic and clinical data $(n=137)$

\begin{tabular}{lcl}
\hline & Mean (SD) & Range \\
\hline Age (years) & $52.0(13.4)$ & 19 to 81 \\
Mean intraocular pressure (mm Hg) & $14.4(3.7)$ & \\
Spherical equivalent (D) & $-1.03(2.08)$ & -5.75 to 2.125 \\
Mean deviation & $0.68(1.04)$ & \\
Pattern SD & $1.64(0.35)$ & \\
\hline
\end{tabular}

Rate of exceeding the 95th percentile of the normative database The rate of exceeding the 95th percentile of the normative database varied considerably according to clock-hour. In general, the rate was high in the non-temporal region, with the highest value being $13.1 \%$ at 9 o'clock, followed by $10.9 \%$ at 10 o'clock. In the nasal region, the values were around $5 \%$. In terms of quadrant thickness, the rate of exceeding the 95th percentile of the normative database was $11.7 \%$ for the temporal quadrant, $7.3 \%$ for the superior quadrant, $3.7 \%$ for the nasal quadrant, and $8.0 \%$ for the inferior quadrant (table 2). In the line-graph analysis, the rate was generally high in the non-temporal region, with the highest value being $23.4 \%$ at $240-250$ test points, followed by $22.5 \%$ at $0-10$ and $250-256$ test points. In the nasal region, the values were $5-10 \%$ (fig 2 ).

\section{DISCUSSION}

OCT measures the RNFL thickness and provides information on the probability of an abnormality after a comparison with an internal normative database. Any method providing the probability of an abnormality by comparison against a normative database has limitations. A given proportion of normal subjects may be recognised as abnormal at a particular level of confidence, that is, $5 \%$ of normal subjects would be identified abnormal at a $<5 \%$ criteria level in OCT.

In the current study, false-positive findings were observed at the expected rate in the nasal region (ie, about $5 \%$ at the $<5 \%$ level). However, in the other regions, the FPR was much lower than expected, especially at 7, 8 and 11 o'clock. The FPRs were $0 \%$ in those three sectors. This means that the lowest thickness noted in our subjects is still greater than the thickness corresponding to the 5 th percentile in the normative database in those sectors. Furthermore, the rate of exceeding the 95th percentile of the normative database was much higher than $5 \%$ in the non-nasal region. These data together suggest that the RNFL thickness profile in our study population may differ from that of the study groups whose data form the normative database.

The current intrinsic normative database was derived from a measurement of 328 normal subjects ${ }^{12}$ comprising 205 (63\%) Caucasian, 79 (24\%) Hispanic, 27 (8\%) black, and only 11 (3\%) Asian subjects, ${ }^{12}$ while the study subjects in the present study were all Korean. Thus, the present findings indirectly suggest that Asians may have a thicker RNFL in the non-nasal regions compared with people of Caucasian or Hispanic ethnicity. This 
Table 2 Number of eyes (\%) showing a false-positive result following OCT analysis of clock-hour and quadrant retinal nerve fibre layer thicknesses at the $<5 \%$ and $<1 \%$ levels and eyes having a measured value which exceeds the 95th percentile of the normative database

\begin{tabular}{|c|c|c|c|}
\hline \multirow[b]{2}{*}{$\begin{array}{l}\text { Retinal nerve fibre } \\
\text { layer thickness }\end{array}$} & \multicolumn{3}{|l|}{ No. of eyes (\%) } \\
\hline & $\begin{array}{l}\text { False positive at } \\
\text { the }<\mathbf{5} \% \text { level }\end{array}$ & $\begin{array}{l}\text { False positive at } \\
\text { the }<\mathbf{1} \% \text { level }\end{array}$ & $\begin{array}{l}\text { Exceeding the 95th } \\
\text { percentile }\end{array}$ \\
\hline 1 o'clock & $4(2.9)$ & $0(0)$ & $10(7.3)$ \\
\hline 2 o'clock & $7(5.1)$ & $1(0.7)$ & $1(0.7)$ \\
\hline 3 o'clock & $3(2.2)$ & $0(0)$ & $7(5.1)$ \\
\hline 4 o'clock & $6(4.4)$ & $0(0)$ & $6(4.4)$ \\
\hline 5 o'clock & $2(1.5)$ & $0(0)$ & $4(2.9)$ \\
\hline 6 o'clock & $2(1.5)$ & $1(0.7)$ & $7(5.1)$ \\
\hline 7 o'clock & $0(0)$ & $0(0)$ & $11(8.0)$ \\
\hline 8 o'clock & $0(0)$ & $0(0)$ & $9(6.6)$ \\
\hline 9 o'clock & $2(1.5)$ & $0(0)$ & 18 (13.1) \\
\hline 10 o'clock & $1(0.7)$ & $0(0)$ & $15(10.9)$ \\
\hline 11 o'clock & $0(0)$ & $0(0)$ & $4(2.9)$ \\
\hline 12 o'clock & $1(0.7)$ & $0(0)$ & $9(6.6)$ \\
\hline Temporal quadrant & $1(0.7)$ & $0(0)$ & 16 (11.7) \\
\hline Superior quadrant & $0(0)$ & $0(0)$ & $10(7.3)$ \\
\hline Nasal quadrant & $7(5.1)$ & $0(0)$ & $5(3.7)$ \\
\hline Inferior quadrant & $3(2.2)$ & $1(0.7)$ & $11(8.0)$ \\
\hline
\end{tabular}

is in accord with a previous report showing that RNFL thickness differed according to race. ${ }^{20} 21$

The difference between the RNFL thickness profile determined in the present study, and that of the normative database, suggests that OCT data from Asian subjects may be significantly different from data obtained from people of other races. Moreover, the thickness profile differences were particularly marked in the superotemporal and inferotemporal regions where glaucomatous defects commonly occur. Thus, the development of a normative database using information derived exclusively from Asian subjects is needed, to increase the utility of OCT in this population.

In addition, the present data suggest that clinicians using the OCT under current guidelines should be aware that false positives are possible when nasal sector abnormalities are studied in Asian subjects. In contrast, subtle abnormalities identified using OCT, such as those only detectable in the line graph, are very likely to be true RNFL defects when they occur in non-nasal sectors. The FPR was extremely low in these sectors.

To detect a false positive, we set, as a gold standard, a combination of the absence of functional visual field loss, but with structural $\mathrm{ONH}$ and RNFL change. Some may argue that such a gold standard is unsuitable for evaluation of an imaging device which is hoped to have a higher sensitivity for early nerve fibre layer loss. If OCT is indeed more sensitive, the abnormal OCT findings documented here may be true defects not detected by conventional methods. OCT as currently practised, however, detects an abnormality by comparing a patient measurement with information in a normative database compiled from measurements on normal subjects. These normal subjects had no visual field abnormality, and no optic nerve or RNFL abnormality. ${ }^{12}$ The subjects of our present study also fulfil these criteria. Thus, any bias introduced into the present study from our definition of a gold standard goes to the heart of current OCT practice. This is the inherent limitation of OCT.

Our suggestion that RNFL thickness profiles may vary according to ethnicity was derived from the unexpectedly low
FPR observed in non-nasal sectors when OCT was employed. This suggestion is reasonable because, as mentioned earlier, the present study obtains and analyses thickness profiles of subjects who are ophthalmologically equivalent (but not ethnically equivalent) to the subjects used to generate the normative database. However, it is obvious that direct comparison of our RNFL thickness measurements to those in the normative database would be a better method for demonstrating racial differences in RNFL thickness distributions. Further measurements of RNFL thicknesses in healthy persons of various ethnicities are required to confirm possible racial differences in RNFL thickness profiles. Our study highlights the need for such work.

In summary, RNFL thickness assessment of Korean eyes using the Stratus OCT resulted in different FPRs in different peripapillary sectors. These data suggest that RNFL thickness profiles may vary according to ethnicity. For the Asian region in particular, this finding suggests the need for an OCT database comprising exclusively Asian subjects. Until then, clinician awareness of possible ethnic differences in RNFL thickness may assist in the diagnosis of glaucoma using OCT.

Funding: Supported by a grant from the Seoul National University Bundang Hospital Research Fund.

Competing interests: None.

\section{REFERENCES}

1. Harwerth RS, Carter-Dawson L, Shen F, et al. Ganglion cell losses underlying visual field defects from experimental glaucoma. Invest Ophthalmol Vis Sci 1999;40:224250.

2. Sommer A, Katz J, Quigley HA, et al. Clinically detectable nerve fiber atrophy precedes the onset of glaucomatous field loss. Arch Ophthalmol 1991;109:77-83.

3. Kass MA, Heuer DK, Higginbotham EJ, et al. The Ocular Hypertension Treatment Study: a randomized trial determines that topical ocular hypotensive medication delays or prevents the onset of primary open-angle glaucoma. Arch Ophthalmol 2002;120:701-13.

4. Girkin CA, MaGwin G Jr, Long C, et al. Subjective and objective optic nerve assessment in African Americans and Whites. Invest Ophthalmol Vis Sci 2004;45:2272-8.

5. Huang D, Swanson EA, Lin CP, et al. Optical coherence tomography. Science 1991:254:1178-81.

6. Schuman JS, Pedut-Kloizman T, Hertzmark E, et al. Reproducibility of nerve fiber layer thickness measurements using optical coherence tomography. Ophthalmology 1996;103:1889-98

7. Schuman JS, Hee MR, Puliafito CA, et al. Quantification of nerve fiber layer thickness in normal and glaucomatous eyes using optical coherence tomography. Arch Ophthalmol 1995;113:586-96.

8. Bowd C, Weinreb RN, Williams JM, et al. The retinal nerve fiber layer thickness in ocular hypertensive, normal, and glaucomatous eyes with optical coherence tomography. Arch Ophthalmol 2000;118:22-6.

9. Zangwill LM, Bowd C, Berry CC, et al. Discriminating between normal and glaucomatous eyes using the Heidelberg Retina Tomograph, GDx Nerve Fiber Analyzer, and Optical Coherence Tomograph. Arch Ophthalmol 2001;119:985-93.

10. Williams ZY, Schuman JS, Gamell L, et al. Optical coherence tomography measurement of nerve fiber layer thickness and the likelihood of a visual field defect. Am J Ophthalmol 2002;134:538-46.

11. Bowd C, Zangwill LM, Berry CC, et al. Detecting early glaucoma by assessment of retinal nerve fiber layer thickness and visual function. Invest Ophthalmol Vis Sci 2001;42:1993-2003.

12. Patella VM. StratusOCT: Establishment of normative reference values for retinal nerve fiber layer thickness measurements. Dublin, CA: Carl Zeiss Meditec, 2003 http://www.meditec.zeiss.com/C125679E00525939/EmbedTitellntern/ Stratus OCT ndb paper/\$File/czm ndb paper.pdf (accessed 15 Nov 2004).

13. Budenz DL, Michael A, Chang RT, et al. Sensitivity and specificity of the StratusOCT for perimetric glaucoma. Ophthalmology 2005;112:3-9.

14. Jeoung JW, Park KH, Kim TW, et al. Diagnostic ability of optical coherence tomography with a normative database to detect localized retinal nerve fiber layer defects. Ophthalmology 2005;112:2157-63.

15. Katz J, Sommer A, Gaarsterland DE, et al. Comparison of analytic algorithm for detecting glaucomatous visual field loss. Arch Ophthalmol 1991;109:1684-9.

16. A nderson DR, Patella VM. Automated static perimetry. 2nd edn. St. Louis: Mosby, 1999:121-90.

17. Swanson EA, Izatt JA, Hee MR, et al. In-vivo retinal imaging by optical coherence tomography. Opt Lett 1993;18:1864-6. 
18. Hee MR, lzatt JA, Swanson EA, et al. Optical coherence tomography of the human retina. Arch Ophthalmol 1995;113:325-32.

19. Hwang JM, Kim TW, Park KH, et al. Correlation between topographic profiles of localized retinal nerve fiber layer defects as determined by optical coherence tomography and red-free fundus photography. J Glaucoma 2006;15:223-8.
20. Poinoosawmy D, Fontana L, Wu JX, et al. Variation of nerve fibre layer thickness measurements with age and ethnicity by scanning laser polarimetry. Br J Ophthalmol 1997:81:350-4.

21. Tsai CS, Zangwill L, Gonzalez C, et al. Racial differences in optic nerve head topography. J Glaucoma 1995;4:248-57.

\title{
Cover illustration
}

\section{The Seated Scribe 2600-2350 BC. The old Kingdom, 4th Dynasty. From Saqquara, north of the valley of the sphinzes, Serapeum, Egypt}

\author{
Credit: Erich Lessing/akg-images
}

This is a painted limestone statue of a scribe portrayed at work. Sons of Didufri (4th Dynasty) were represented in this seated position, so it is thought that this may represent a member of the royal family. The most remarkable aspect about the statue is the face, in particular the eyes. They consist of a piece of red-veined white magnesite, in which a piece of slightly truncated rock crystal was placed. The front part of the crystal was carefully polished. The back side was covered with a layer of organic material, creating the colour of the iris and also probably serving as an adhesive. The entire eye was then held in the socket by two large copper clips welded on the back. A line of black paint defines the eyebrows. The hands, fingers, and fingernails are sculpted with a remarkable delicacy. When viewed first-hand (at the Louvre, Paris), the eyes look like they were only recently minted. A modern ocularist would be proud to be the designer of such prosthetic eyes.

As with most things that evolved over time, it is difficult to trace the inventor of the artificial glass eye used in humans, but William Shakespeare (1564-1616) knew of its existence: "Get thee glass eyes;/And, like a scurvy politician, seem/To see the things thou dost not." (King Lear to the Earl of Gloucester, Act IV, Scene 6).

It has been suggested that the ancient Egyptians and Indians knew how to fashion prosthetic eyes. Whereas the Seated Scribe does not confirm that they used these remarkable prosthetic eyes in humans as well, it certainly shows their ingenuity and suggests possibilities. This statue may be the oldest example of three-dimensional eyes.

\section{Bhupendra C K Patel, Arun Singh, Harminder Dua}

